\title{
Industry Perspective on Dissolution Apparatus Galibration
}

Timothy J. MeCormiek The DuPont Merck Pbarmaceutical Company, Wilmington, DE

\section{Abstract}

The USP dissolution apparatus suitability test procedure using USP calibrator tablets is currently a controversial topic within the pharmaceutical industry. In order to develop an understanding of the industry's perspective on dissolution calibration, a survey was conducted in the pharmaceutical industry on this subject. This survey has provided the industry with an opportunity to discuss the issues surrounding dissolution calibration and to suggest options for improvements. The results of the survey are presented delineating the industry's concerns and offering alternatives to the current dissolution calibration procedure.

\section{Intraduction}

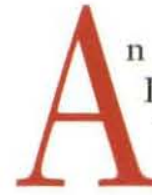

n AAPS/USP workshop on Dissolution Calibration and Testing was held on September 27-28, 1995, in Arlington, Virginia. At this workshop, I presented the results of an industry survey on dissolution apparatus calibration in a talk titled "Industry Perspective - Issues and Difficulties with Dissolution Calibration." The survey provided the industry with an opportunity to express their concerns regarding dissolution calibration and to suggest options for improvements. It also allowed me to gain a good understanding of the industry's perspective on dissolution calibration and to accurately present this at the workshop. This article summarizes the more important results of the survey as presented at the workshop.

A survey consisting of 10 open-ended questions was sent to 93 organizations representing industry, government, and equipment manufacturers. The organizations were selected from members of the National Stability Discussion Group and from readers of Dissolution Technologies. Thirty-five responses were received from 29 organizations, representing a $38 \%$ response rate. Thirty-four responses were usable since one respondent did not conduct dissolution testing. The results from these 34 respondents were the basis for the workshop presentation and for this article.
An abbreviated version of the survey (i.e. six questions) was published in the August 1995 issue of Dissolution Technologies (Volume 2, Issue 3). A review of these additional responses indicates that they are consistent with the overall results of the survey.

\section{Results and Discussion}

The majority of the respondents $(72 \%)$ calibrate their dissolution apparatus on a semiannual basis. The most common reason given for using a 6-month calibration frequency was that it is an SOP/company requirement. Other reasons include: FDA requirement, GMP compliance, USP recommendation, and "industry standard." Although no compendial/regulatory requirements exist for semiannual calibration $(1,2)$, this frequency has been recommended by several authorities in the field $(3,4)$. In addition, various practices were reported both within and across companies in regard to calibration frequency. For example, some companies reported running calibration tablets semiannually, but mechanical checks annually and vice versa. Also, different practices were reported between departments within a company such as one department calibrating semiannually and another annually.

Nearly one-third (30\%) of the responses regarding the pur- 
pose of calibration indicated that the reason for calibration of a dissolution apparatus was to ensure proper operation/performance of the system. However, half of the responses stated that calibration is only performed either because it is a USP requirement $(28 \%)$ or to comply with GMP's (22\%). The feeling is that there is not much value in these calibration tests. That is, the calibrators do not ensure accurate performance of the dissolution apparatus. It is felt that physical/mechanical parameters are more important in terms of ensuring accurate performance.

When asked if USP calibrator tablets are necessary/useful for dissolution apparatus calibration, the most frequent responses were yes $(27 \%)$ they are necessary, but no (33\%) they are not useful. The most important reason cited as to why the tablets are necessary was to assess the overall performance of the dissolution system. The main reason cited for why the tablets are not useful is because of too much tablet variability. In short, the thinking is that calibrator tablets are necessary, but the current USP calibrators are not useful. Failures are usually due to the tablets themselves, not the alignment of the apparatus. In order to justify the huge expenditure of time necessary to perform a calibration; development of more reliable, reproducible, and sensitive calibrators was recommended.

In regard to recommenda- tions for alternatives and/or more suitable procedures for calibration other than using calibrator tablets, over one-third (41\%) of the responses recommended exclusively using and/or tightening mechanical parameters. Another one-third $(33 \%)$ of the responses did not contain any specific suggestions, but indicated that analysts would like to see: less time needed for calibration, discontinued use of calibrator tablets, and calibrator tablets that are more applicable. Fifteen percent of the responses suggested the use of an in-house product for calibration. In addition, it was noted that: more sensitive calibrator tablets are needed, a film-coated tablet could be used to reduce moisture susceptibility and tablet friability, and flask uniformity needs to be improved and specifications tightened.

As for changes and/or improvements to the USP calibration procedure, the most frequently recommended change $(21 \%)$ was to replace current USP calibrators with: more sensitive tablets, calibrators with better uniformity, and tablets that are not influenced by extraneous factors. Other important recommendations were to: use/tighten mechanical specifications $(17 \%)$, reduce testing $(14 \%)$, and provide retesting criteria $(7 \%)$. In addition, some of the less frequent responses included: specifying levels of deaeration (e.g. oxygen readings), conducting more inter-lab testing prior to release of batches /specifications, and providing a less time consuming USP procedure for degassing media. Specific recommendations regarding tighter mechanical specifications were as follows: centering \pm 1 $\mathrm{mm}, \mathrm{RPM} \pm 2 \%$, wobble NMT $1 \mathrm{~mm}$, set specifications on tilt angle and vibration, and tighten flask specifications. In addition, specific recommendations for reduced testing were to: eliminate the use of two sets of tablets, use salicylic acid only with baskets and prednisone only with paddles, and use only one rotational speed.

Slightly less than one-third $(31 \%)$ of the respondents calibrate for both apparatus 1 and 2 since they use both methods frequently. Almost another onethird $(31 \%)$ said they only calibrate for one apparatus, usually paddles, since basket methods are not used. According to Gray et al. (4), the practice of only calibrating the apparatus you are using is acceptable. Over onethird $(37 \%)$ do a combination of one apparatus and both apparatuses. Typically, most dissolution baths in a laboratory would be calibrated for paddles and a few would be calibrated for both paddles and baskets.

A large majority of the respondents (94\%) have had difficulty meeting the USP calibrator tablet specifications. Almost half $(48 \%)$ indicated problems with prednisone tablets. Onethird (33\%) said they had difficulties with both salicylic acid and prednisone tablets, whereas 


\section{Industry Perspective....cont.}

only $6 \%$ had problems with salicylic acid tablets alone. Both apparatuses at both speeds (50 and $100 \mathrm{rpm}$ ) were reported to be problematic. There was no apparent trend for any particular apparatus or speed. Although failures occur with both prednisone and salicylic acid tablets on both apparatuses and at both speeds, it seems that usually nothing ever proves to be "wrong" with the bath.

Actually, in response to a question concerning the nature of the problem, the largest problem reported $(61 \%)$ was "failing results" for no apparent reason. Other problems reported were: calibrator tablet variability (10.5\%), defective condition of the tablets $(10.5 \%)$, insufficient/improper deaeration ( $8 \%$ ), and physical/ mechanical problems (5\%). The most common solution reported $(27 \%)$ for problem resolution was to retest until passing results are obtained. Other important solutions reported were: change deaeration technique/ensure proper deaeration (19\%), use new bottle/lot of tablets (17\%), and verify physical/mechanical parameters (15\%).

Over two-thirds (71\%) of the respondents said they never had a case where calibration failure was related to a problem with the dissolution apparatus itself such as poor alignment, etc. This is apparently due to the fact that respondents typi- cally ensure that the apparatus passes all physical parameters before running the calibrator tablets. Of those who have had a case of failure related to the apparatus itself (29\%), most of the apparatus problems encountered could be attributed to vibration (caused by worn bearings, worn drive belts, immersion heaters, and other equipment located nearby) which is consistent with the fact that calibrator tablets are sensitive to vibration $(4,5)$.

Similarly, over two-thirds $(69 \%)$ of the respondents said they never had anomalous and/or variable product results that were related to a problem with the dissolution apparatus itself. Of those who have stated they have experienced such a case $(17 \%)$, only one-third said the apparatus was meeting calibration specifications whereas the other two-thirds either did not know or gave no response. The low occurrence of questionable product results attributable to the dissolution apparatus itself may be related to the above cited practice of checking the physical/mechanical parameters before running a dissolution test. This practice may be more beneficial in ensuring the quality of product data than running the calibrator tablets themselves.

\section{Conclusion}

Most analysts feel that a calibrator tablet is necessaary, but the current USP calibrators are not adequately serving the purpose of ensuring proper performance of the dissolution system. They would like to see the uniformity of the calibrator tablets improved, and the tablets be made more sensitive to physical parameters and less sensitive to extraneous factors. In addition, mechanical parameters are very important and tolerances should be tightened.

The number of calibration tests should be reduced to a maximum of two tests, one for the paddle apparatus and one for the basket apparatus. This should be sufficient to gain information on the performance of each type of apparatus. For example, prednisone tablets could be used with the paddle apparatus at $50 \mathrm{rpm}$ since prednisone is a disintegrating tablet and this is the most appropriate apparatus and speed for such a tablet. In a similar fashion, salicylic acid tablets could be used with baskets at $100 \mathrm{rpm}$ since this is a non-disintegrating tablet for which baskets are recommended (6). This would greatly reduce calibration testing from eight tests taking 2-3 days to perform to two tests taking 0.5 1 day. Also, if only one apparatus is being used then calibration testing can be reduced to one test taking about a half-day to run. Additionally, consideration should be given to using only one tablet type. The nondisintegrating tablet type could be eliminated since this is not a 
frequently encountered dosage form in the pharmaceutical industry and is of little relevance to the majority of dissolution testing being conducted industry-wide.

Finally, retesting criteria should be provided for cases of failure when there is nothing proven to be wrong with the system. Apparently, most failures are due to the variability of the tablets themselves rather than problems with the apparatus. In addition, the deaeration issue needs to be addressed. Since the USP recommended manual deaeration technique $(1,4)$ is too cumbersome and inefficient for preparing large volumes of deaerated medium, most analysts use alternate methods (e.g., helium sparging, automated equipment, etc.) that are more efficient for large volumes. However, calibrator tablets should perform similarly in sufficiently deaerated medium regardless of the method used to deaerate the medium. Therefore, a specification on deaeration needs to be established that is independent of the deaeration technique. For instance, Hanson (7) recommends that the dissolved oxygen in the medium be at least $5 \%$ below the saturation value at $37^{\circ} \mathrm{C}$. This will help to prevent the release of dissolved gases in the dissolution medium during the test.

\section{Acknowledgements}

I am thankful to

Kim Huynh-Ba and Cynthia Brown for providing me with contacts from the National Stability Discussion Group and Dissolution Technologies respectively. Also, I am grateful to all the respondents who took the time and effort to respond to the survey.

\section{Mefterenters}

(1) USP $23 / \mathrm{NF} 18$; United States Pharmacopeial Convention; Rand McNally: Taunton, 1994; pp 1791- 1793.

(2) "Current Good Manufacturing Practice for Finished Pharmaceuticals (CGMPRs)"; Part 211.160(b)(4); United States Food and Drug Administration; Handi-Regs \#8001, Keystone: Bradenton, 1995; p 42.

(3) Hanson, W. A. Handbook of Dissolution Testing; Aster: Eugene, 1991; p 109.

(4) Gray, V. A.; Hubert, B. B.; Krasowski, J. A. Pharm Forum 1994, 20 (6), 8571-8573.

(5) Grady, L. T. Pharm Forum 1994, 20 (6), 8567-8570.

(6) USP 23/NF 18; United States Pharmacopeial Convention; Rand McNally: Taunton, 1994; p 1925.
(7) Hanson, W. A. Handbook of Dissolution Testing; Aster: Eugene, 1991; p 83. 